\title{
A Lyapunov Theorem for Angular Cones
}

\author{
Charles R. Johnson* \\ Institute for Basic Standards, National Bureau of Standards, Washington, D.C. 20234
}

(August 9, 1973)

\begin{abstract}
The well known theorem of Lyapunov is generalized to characterize matrices whose spectra lie in a given open convex angular sector. Related facts about positive definite matrices, the polar decomposition and matrices with cramped spectra are also given.
\end{abstract}

Key words: Cramped; field of values; open positive convex cone; polar decomposition; positive definite; spectrum; square matrix.

A theorem of Lyapunov which has several equivalent formulations characterizes the matrices whose eigenvalues lie in the right half-plane. By considering one of the formulations from a new point of view, a characterization of the matrices whose eigenvalues lie in an arbitrary open positive convex cone is obtained. The positive cone of the characterization generalizes the right half-plane of Lyapunov's Theorem.

Throughout we shall consider square matrices $A, B, C \ldots$ over the complex field. Denote the field of values of $A$ by $F(A)=\left\{x^{*} A x \mid\|x\|=1\right\}$ and the set of eigenvalues (spectrum) of $A$ by $\sigma(A)$. It is well known that $F(A)$ is a compact convex subset of the complex plane which contains $\sigma(A)$ and that $F(A)$ coincides with the convex hull of $\sigma(A)$ when $A$ is normal. We shall also employ the concept of the angular field $F_{\text {ang }}(A)=\left\{x^{*} A x \mid x \neq 0\right\}$ mentioned by Wielandt [5] ${ }^{1}$ and the angular spectrum $\sigma_{\text {ang }}(A)=\left\{\sum_{i=1}^{n} \alpha_{i} \lambda_{i} \mid \sigma(A)=\left\{\lambda_{1}, \ldots, \lambda_{n}\right\} ; \alpha_{i} \geqslant 0, i=1, \ldots, n ;\right.$ and $\left.\sum_{i=1}^{n} \alpha_{i}>0\right\}$. $F_{\text {ang }}(A)$ and $\sigma_{\text {ang }}(A)$, respectively, are just the smallest positive convex cones which are anchored at the origin and contain $F(A)$ and $\sigma(A)$. Finally let $R$ signify the open right complex half-plane and $\Sigma$ be the class of positive definite hermitian matrices.

The three versions of Lyapunov's Theorem of interest are

(1) [Strong Form [3]]

$\sigma(A) \subset R$ if and only if for each $H \epsilon \Sigma$ there is a $G \epsilon \Sigma$ such that $G A+A^{*} G=H$;

(2) $[$ Weak Form $]$

$\sigma(A) \subset R$ if and only if there is a $G \epsilon \Sigma$ such that $G A+A^{*} G \epsilon \Sigma$; and

(3) [Field of Values Formulation]

$\sigma(A) \subset R$ if and only if there is a $G \epsilon \Sigma$ such that $F(G A) \subset R$.

That (2) and (3) are equivalent follows immediately from the fact that the field of values of $\frac{1}{2}\left(G A+A^{*} G\right)$ is just the projection of $F(G A)$ onto the real axis. For a discussion of the equivalence of (1) and (2) see $[3,4]$.

AMS Subject Classification: 15-A18,-A42, -A57, -A63.

${ }^{*}$ This work was done while the author was a National Academy of Sciences-National Research Council Postdoctoral Research Associate at the National Bureau of Standards, Washington, D.C. 20234.

${ }^{1}$ Figures in brackets indicate the literature references at the end of this paper. 
Formulation (3) is of interest in that it points out that Lyapunov's Theorem relates a region of containment for $\sigma(A)$ to a region of containment for the field of values of a positive definite multiple of $A$. In particular the two conic regions of containment are the same. We shall show that the $R$ of Lyapunov's theorem (3) can be replaced by an arbitrary open positive convex cone $\Gamma$ ( $\Gamma$ shall be such throughout). This then characterizes $\{A \mid \sigma(A) \subset \Gamma\}$ and relates the eigenvalues and field of values for arbitrary matrices. It should be noted that the following is of interest only when $\Gamma$ describes an are of less than or equal to $\pi$.

Theorem A: The set $\sigma(\mathrm{A}) \subset \Gamma$ if and only if there is $a \mathrm{G} \epsilon \Sigma$ such that $\mathrm{F}(\mathrm{GA}) \subset \Gamma$.

We present the proof of the theorem using 3 lemmas.

Let $C o$ denote the convex closure and $d$ the distance function.

LEMMA 1: Given an arbitrary square complex matrix A and a positive number $\epsilon$, there exists an invertible matrix $\mathrm{S}$ such that

$$
\mathrm{d}\left(\mathrm{F}\left(\mathrm{SAS}^{-1}\right), \operatorname{Co}(\sigma(\mathrm{A}))\right)<\epsilon
$$

(The field of values can be made close to the convex hull of the spectrum via similarity.)

Proof: Reduce $A$ to Jordan form $J$ by similarity. If $J$ is diagonal, we are done. If not, the superdiagonal l's may be made arbitrarily small by diagonal similarities. Thus $F\left(D^{-1} J D\right)$ may be made arbitrarily close to the convex hull of the diagonal of $J$ which is just $\operatorname{Co}(\sigma(A))$.

LEMMA 2: Let $\operatorname{det} \mathrm{B} \neq 0$. Then

$$
\mathrm{F}_{\text {ang }}\left(\mathrm{B}^{*} \mathrm{AB}\right)=\mathrm{F}_{\text {ang }}(\mathrm{A}) \text {. }
$$

(The invariance of the angular field under congruence will mean that $F\left(B^{*} A B\right) \subset \Gamma$ if and only if $F(A) \subset \Gamma$.)

Proof: $x^{*} B^{*} A B x=y^{*} A y$ and $y=B x \neq 0$ if and only if $x \neq 0$.

LEMMA 3: If $\mathrm{G} \epsilon \Sigma$, then

$$
\sigma(\mathrm{A}) \subset \sigma_{\text {ang }}(\mathrm{A}) \subset \mathrm{F}_{\text {ang }}(\mathrm{GA})
$$

Proof: It suffices to show $\sigma(A) \subset F_{\text {ang }}(G A)$. Since $G \epsilon \Sigma, G$ may be written $G=P^{*} P$, $\operatorname{det} P \neq 0$. Then,

$$
\sigma(A)=\sigma\left(P A P^{-1}\right)=\sigma\left(\left(P^{-1}\right) * G A P^{-1}\right) \subset F\left(\left(P^{-1}\right) * G A P^{-1}\right) \subset F_{\text {ang }}\left(\left(P^{-1}\right) * G A P^{-1}\right)=F_{\text {ang }}(G A) .
$$

Proof (of theorem): Suppose $\sigma(A) \subset \Gamma$; choose $S$ by lemma 1 so that

$$
d\left(F\left(S A S^{-1}\right), \operatorname{Co}(\sigma(A))\right)<\min _{c \in \bar{\Gamma}}(\operatorname{Co}(\sigma(A)), c)
$$

which is positive. This means $F\left(S A S^{-1}\right) \subset \Gamma$. By lemma $2, F\left(S^{*}\left(S A S^{-1}\right) S\right) \subset \Gamma$ also, so that we have $F(G A) \subset \Gamma$ where $G=S * S \epsilon \Sigma$.

For the converse suppose $F(G A) \subset \Gamma, G \epsilon \Sigma$. Then $F_{\text {ang }}(G A) \subset \Gamma$ and by lemma 3 , $\sigma(A) \subset F_{\text {ang }}(G A) \subset \Gamma$ and the theorem is proven.

That $\Sigma$ is the only class of matrices enjoying the property of lemma 3 is of individual interest. Theorem B: $\sigma(\mathrm{A}) \subset \mathrm{F}_{\text {ang }}(\mathrm{GA})$ for all matrices $\mathrm{A}$ if and only if $\mathrm{G} \epsilon \Sigma$.

Proof: It suffices to show that $\sigma(A) \subset F_{\text {ang }}(G A)$ for all $A$ implies $G \epsilon \Sigma$ since the converse has been proven in lemma 3 . 
First let $A$ run through $\Sigma$ and notice that $\sigma\left(A G^{*}\right) \subset F_{\text {ang }}\left(G A G^{*}\right)=F_{\text {ang }}(A)=$ the positive real axis. This means that $\sigma(G A)$ is real and positive for all $A \epsilon \Sigma$. Now write $G$ in its polar form $G=U H$ where $U$ is unitary and $H \epsilon \Sigma$. We may take $A=H^{-1} \epsilon \Sigma$ and then $G A=U H H^{-1}=U$. Since $\sigma(U)=\sigma(G A)$ is real and positive, $U$ can only be the identity. Thus $G=H \epsilon \Sigma$.

Several remarks now follow from the preceding.

Corollary 1: $\sigma_{\text {ang }}(\mathrm{A})=\bigcap_{\mathrm{G} \in \Sigma} \mathrm{F}_{\text {ang }}(\mathrm{GA})$.

Proof: By theorem $\mathrm{B} \sigma_{\text {ang }}(A) \subset \bigcap_{\mathrm{G} \in \Sigma} F_{\text {ang }}(G A)$; but in theorem $\mathrm{A}$ we may choose $\Gamma$ as close as we like to $\sigma_{\text {ang }}(A)$ which proves the equality.

Corollary 2: If $\mathrm{A}^{*} \mathrm{C} \epsilon \Sigma$ (or equivalently $\mathrm{CA}^{-1} \epsilon \Sigma$ ), then $\sigma(\mathrm{A}) \subset \mathrm{F}_{\text {ang }}(\mathrm{C})$.

Proof: Suppose $C A^{-1}=H \epsilon \Sigma$, then

$$
\sigma(A) \subset F_{\text {ang }}(H A)=F_{\text {ang }}(C) .
$$

Corollary 3: Suppose $\mathrm{A}=\mathrm{HU}$, where $\mathrm{U}$ is unitary and $\mathrm{H} \epsilon \Sigma$ is the polar decomposition of $\mathrm{A}$, then

$$
\sigma_{\text {ang }}(A) \subset F_{\text {ang }}(U) \subset F_{\text {ang }}(A) .
$$

Proof: By assumption $A U^{*}=H \epsilon \Sigma$ or, equivalently, $U A^{-1} \epsilon \Sigma$ which implies $\sigma_{\text {ang }}(A) \subset F_{\text {ang }}(U)$ by corollary 2 . Since $U$ is normal, $F_{\text {ang }}(U)=\sigma_{\text {ang }}(U)$ which is contained in $F_{\text {ang }}(H U)=F_{\text {ang }}(A)$ by Theorem B.

In order to characterize the matrices whose eigenvalues lie in a half-plane, we define a matrix $A$ to be cramped if (1) det $A \neq 0$ and (2) the set $\left\{\frac{\lambda}{|\lambda|} \mid \lambda \epsilon \sigma(A)\right\}$ lies on an arc of the unit circle of less than $180^{\circ}$. It is clear that this is equivalent to saying the maximum pairwise difference of the arguments of the eigenvalues is less than $\pi$ (and $0 \notin \sigma(A))$ or there is a $\theta$ such that $\sigma\left(\mathrm{e}^{i \theta} A\right) \subset R$. We then have

COROLLARY 4: If det $\mathrm{A} \neq 0$, the following statements are equivalent:

(i) A is cramped;

(ii) $0 \notin \sigma_{\text {ang }}(\mathrm{A})$;

(iii) there is a $\mathrm{G} \epsilon \Sigma$ such that $0 \notin \mathrm{F}(\mathrm{GA})$;

(iv) there is a $\mathrm{G} \epsilon \Sigma$ such that $\mathrm{F}_{\text {ang }}(\mathrm{GA})$ is not the entire complex plane and $\mathrm{A}$ does not have two characteristic roots which determine a line segment containing 0 .

Proof: The equivalence of (i) and (ii) is immediate and the equivalence of (i) and (iii) follows from theorem A. If $F_{\text {ang }}(G A)$ is not the entire complex plane, it can be at most a closed half-plane which means $\sigma_{\text {ang }}(A)$ is at most a closed half-plane by theorem B. It then follows that (iv) implies (i). Since $0 \notin F(G A)$ implies $0 \notin F_{\text {ang }}(G A)$ and that $A$ is cramped, we have that (iii) implies (iv) and the corollary is complete.

COROLLARY 5: If $\mathrm{G} \epsilon \Sigma$ and $\mathrm{GA}$ is hermitian (resp. hermitian positive definite), then the roots of $\mathrm{A}$ are real (resp. real and positive).

Proof: An application of Corollary 2. This last corollary is known by other means: see [2, 5].

In order to facilitate a final corollary which translates theorem $\mathrm{A}$ into a representation theorem, let $F(\Gamma)=\{A \mid F(A) \subset \Gamma\}$ and $S(\Gamma)=\{A \mid \sigma(A) \subset \Gamma\}$ where $\Gamma$ is as before. It is clear that $F(\Gamma)$ $\subset S(\Gamma)$.

Corollary 6. $\mathrm{S}(\Gamma)=\Sigma \cdot \mathrm{F}(\Gamma)$

Proof: Suppose $A \epsilon \Sigma \cdot F(\Gamma)$, then $A=P B$ where $P \epsilon \Sigma$ and $F(B) \subset \Gamma$. Then $P^{-1} \epsilon \Sigma$ and $P^{-1} A$ $=B \epsilon F(\Gamma)$ which implies $\sigma(A) \subset \Gamma$ by theorem $\mathrm{A}$ and means $A \epsilon S(\Gamma)$.

If $A \epsilon S(\Gamma)$, then also by theorem A, we have $F(G A) \subset \Gamma$ for some $G \epsilon \Sigma$. Since $G^{-1} \epsilon \Sigma$ and $A=G^{-1}(G A)$ we have $A \epsilon \mathcal{L}^{\prime} \cdot F^{\prime}(\Gamma)$ to complete the proof.

Finally one question is suggested and left for further study by theorem A. How may we characterize the $B$ 's such that $F(B) \subset \Gamma, B=G A$ where $G \epsilon \Sigma$ and $\sigma(A) \subset \Gamma$, or alternatively how do 
we characterize the $G$ 's in $\Sigma$ such that $F(G A) \subset \Gamma$. When $\Gamma=R$, this question is answered in part by Lyapunov's theorem (1) and the invertibility of the Lyapunov operator. In this case any positive definitite real part may be obtained, but with each real part only one imaginary part may be obtained. Thus many $B$ with $F(B) \subset R$ are omitted from the range.

\section{References}

[1] Givens, W., Fields of values of a matrix, Proc. AMS 3, 206-209 (1952).

[2] Taussky-Todd, O., Problem 4846, Am. Math. Monthly 66, 427 (1959).

[3] Taussky-Todd, O., Matrices $C$ with $C^{n} \rightarrow 0$, J. of Algebra 1,5-10 (1964).

[4] Taussky-Todd, O., On stable matrices, Colloques Internationaux Du Centre National De La Recherche Scientifique No. 165; Programmation En Mathematiques Numeriques, Sept. 1966.

[5] Wielandt, H., On the eigenvalues of $A+B$ and AB, J. Res. Nat. Bur. Stand. (U.S.), 77B (Math. Sci.) Nos. 1 \& $2,61-63$ (Jan.-June 1973).

[6] Wigner, E. P., On weakly positive matrices, Can. J. of Math. 15, 313-317 (1963).

[7] Williams, J. P., Similarity and the numerical range, J. Math. Anal. and Applics. 26, 307-314 (1969).

(Paper 78B1-393) 